\title{
PARK20 caused by SYNJ1 homozygous Arg258GIn mutation in a new Italian family
}

\author{
Simone Olgiati • Anna De Rosa - Marialuisa Quadri • Chiara Criscuolo • \\ Guido J. Breedveld • Marina Picillo • Sabina Pappatà • Mario Quarantelli • \\ Paolo Barone • Giuseppe De Michele • Vincenzo Bonifati
}

Received: 24 April 2014 / Accepted: 25 April 2014 / Published online: 10 May 2014

(C) Springer-Verlag Berlin Heidelberg 2014

\begin{abstract}
SYNJ1 has been recently identified by two independent groups as the gene defective in a novel form of autosomal recessive, early-onset atypical parkinsonism (PARK20). Two consanguineous families were initially reported (one of Sicilian and one of Iranian origins), with the same SYNJ1 homozygous mutation (c.773G $>$ A, p.Arg258Gln) segregating with a similar phenotype of earlyonset parkinsonism and additional atypical features. Here, we report the identification of the same SYNJ1 homozygous
\end{abstract}

Simone Olgiati and Anna De Rosa contributed equally to this work and should be considered as joint first authors.

Giuseppe De Michele and Vincenzo Bonifati contributed equally to this work and should be considered as joint senior authors.

Electronic supplementary material The online version of this article (doi:10.1007/s10048-014-0406-0) contains supplementary material, which is available to authorized users.

S. Olgiati · M. Quadri · G. J. Breedveld • V. Bonifati $(\square)$

Department of Clinical Genetics, Erasmus MC, PO Box 2040,

3000 CA Rotterdam, The Netherlands

e-mail: v.bonifati@erasmusmc.nl

\author{
A. De Rosa $\cdot$ C. Criscuolo • M. Picillo · G. De Michele \\ Department of Neurosciences and Reproductive and \\ Odontostomatological Sciences, "Federico II" University, \\ Naples, Italy \\ S. Pappatà • M. Quarantelli \\ Biostructure and Bioimaging Institute, National Research Council, \\ Naples, Italy \\ P. Barone \\ Department of Medicine and Surgery, CEMAND, University of \\ Salerno, Salerno, Italy \\ G. De Michele $(\square)$ \\ Dipartimento di Neuroscienze, Scienze Riproduttive ed \\ Odontostomatologiche, Università degli Studi di Napoli Federico II, \\ Via Pansini 5, 80131 Naples, Italy \\ e-mail: demichel@unina.it
}

mutation in two affected siblings of a third pedigree. Both siblings had mild developmental psychomotor delay, followed, during the third decade of life, by progressive parkinsonism, dystonia, and mild cognitive impairment. One sibling suffered one episode of generalized seizures. Neuroimaging studies revealed severe nigrostriatal dopaminergic defects, mild striatal and very mild cortical hypometabolism. Treatment with dopamine agonists and anticholinergics resulted in partial improvements. Genetic analyses revealed in both siblings the SYNJ1 homozygous c.773G $>$ A (p.Arg258Gln) mutation. Haplotype analysis suggests that the mutation has arisen independently in this family and the Sicilian PARK20 family previously described by us, in keeping with the hypothesis of a mutational hot spot. This is the third reported family with autosomal recessive, early-onset parkinsonism associated with the SYNJ1 p.Arg258Gln mutation. This work contributes to the definition of the genetic and clinical aspects of PARK20. This newly recognized form must be considered in the diagnostic work-up of patients with early-onset atypical parkinsonism. The presence of seizures might represent a red flag to suspect PARK20.

Keywords PARK20 - SYNJ1 · Synaptojanin 1 .

Parkinsonism $\cdot$ Mutation $\cdot$ Parkinson's disease

\section{Introduction}

The autosomal recessive, early-onset parkinsonism is highly heterogeneous on both clinical and genetic levels. Mutations in parkin, PINK1, and DJ-1 cause parkinsonism with a good and prolonged response to dopaminergic therapy, and clinically presenting as early-onset Parkinson's disease. On the contrary, mutations in ATP13A2, PLA2G6, FBXO7, and $D N A J C 6$ cause early-onset atypical parkinsonism, with poor response to levodopa and additional clinical signs 
(oculomotor disturbances, dystonia, pyramidal signs, cognitive decline) of variable severity [1].

SYNJ1 has been recently identified by us [2] and by an independent group [3] as the gene defective in a novel form of autosomal recessive, early-onset atypical parkinsonism. Two consanguineous families were initially reported (one of Sicilian and one of Iranian origins), with the same SYNJ1 homozygous c.773G $>$ A mutation (p.Arg258Gln) segregating with early-onset atypical parkinsonism. This form has been designated as PARK20 (Online Mendelian Inheritance in Man, OMIM, 615530). The SYNJ1 gene encodes synaptojanin-1, a phosphoinositide phosphatase protein involved in the regulation of post-endocytic recycling of synaptic vesicles. Here, we report the identification of the same SYNJ1 homozygous mutation in two affected siblings of a third pedigree, originating from the Campania region in Southern Italy.

\section{Materials and methods}

This study was approved by the appropriate institutional review boards. Written informed consent was obtained from all participants.

Clinical studies We studied one Italian pedigree originating from Campania (Southern Italy) with two out of four siblings affected by early-onset, atypical parkinsonism. There was no known parental consanguinity (the parents originate from the same district of Naples, where $\sim 100,000$ inhabitants are residing) and no history of neurological diseases in the previous generations. We performed detailed neurological and neuropsychological evaluations, including: mini mental state examination (MMSE); Raven's Coloured Progressive Matrices, for the assessment of general intelligence and visuospatial abilities; Spatial Span, for spatial short-term memory; Verbal Span, for verbal short-term memory; Rey's 15-word immediate and delayed recall and story recall, for verbal long-term memory; Arrigoni-De Renzi test, for constructional skills; and Frontal Assessment Battery (FAB), attentive matrices, and phonemic verbal fluency test, for attention and executive functions.

Neuroimaging studies Brain MRI studies were carried out at 3 T (TRIO, Siemens Medical Solutions, Erlangen, Germany) in one patient, acquiring 3D T1-weighted Gradient-echo volume with isotropic voxels, axial diffusion-weighted images, and axial and coronal $\mathrm{T} 2$-weighted turbo spin-echo and fluidattenuated inversion recovery (FLAIR) images. In the other patient, MRI was performed at $1.5 \mathrm{~T}$ (Achieva, Philips Healthcare, Eindhoven, NL) acquiring axial conventional spin-echo T1-weighted images, axial diffusion-weighted and fluid-attenuated inversion recovery (FLAIR) images, and sagittal and coronal T2-weighted turbo spin-echo images.
MRI images ( $3 \mathrm{~T}$ ) obtained with the same protocols in the patients of the previous family with SYNJ1 mutations previously described by us [2] were also available for comparison.

Brain SPECT studies were performed $4 \mathrm{~h}$ after an intravenous injection of $185 \mathrm{MBq}$ of $\left[{ }^{123} \mathrm{I}\right] \mathrm{FP}-\mathrm{CIT}$ (DaT-SCAN, GEHealthcare, Wauwatosa, WI), and thyroid blockade with oral administration of Lugol's solution. SPECT was performed using a dual-headed camera (E. CAM, Siemens Medical Systems, Hoffman Estates, IL). Images were reconstructed using a Butterworth filter (cut-off 0.5, order 10) and corrected for attenuation using Chang's algorithm $\left(\mu=0.06 \mathrm{~cm}^{-1}\right)$. Region of interest (ROI) analysis was performed using a template of circular ROIs for caudate and putamen of right and left hemispheres and a polygonal ROI for the occipital cortex [4]. The outcome measure was the specific-to-nondisplaceable binding ratio, V3" (ROI striatum-ROI occipital/ROI occipital).

$\left[{ }^{18} \mathrm{~F}\right]$ FDG-PET brain images were acquired for $15 \mathrm{~min}$ in 3D mode between 45 and 60 min after intravenous administration of $213-250 \mathrm{MBq}$ of $\left[{ }^{18} \mathrm{~F}\right]$ fluorodeoxyglucose in resting state and eyes closed condition, using a whole body PET/ CT scanner (Discovery LS, GE Medical System, Milwaukee, WI). Images were reconstructed with iterative reconstruction (FORE-Iterative) and corrected for attenuation using the CT scan. Outcome measure was the relative glucose metabolism. Both SPECT and PET images were spatially normalized in the Montreal Neurological Institute (MNI) space using SPM2 (Wellcome Department of Imaging Neuroscience, London, UK).

Genetic studies Genomic DNA was isolated from peripheral blood with standard protocols. Sanger sequencing and Multiple Ligation Probe Amplification (MLPA) kits P051 and P052 (MRC Holland, Amsterdam, The Netherlands) were used to screen the known genes causing early-onset parkinsonism in the affected family members (sequencing of all exons and exon-intron boundaries of parkin, PINK1, DJ-1, ATP13A2, $P L A 2 G 6, F B X O 7$, and DNAJC6, and exon dosage of SNCA, parkin, PINK1, DJ-1, and ATP13A2). Genome-wide homozygosity mapping was performed using Illumina HumanOmniExpress BeadChip array data (730,525 SNPs at a median distance of $2.1 \mathrm{~kb}$ ). Samples from the two affected siblings (NAPO-41 and NAPO-42) were included. Data were analyzed using Nexus Copy Number, Discovery Edition, Version 7 (BioDiscovery, El Segundo, CA). Direct sequencing of the SYNJ1 gene (MIM 604297) was performed using the Sanger method, and primers and PCR protocols previously described by us [2] (GenBank accession numbers NM_003895.3 and NP_003886.3). Haplotype analysis of the new family and the Sicilian family previously reported by us with the same SYNJ1 mutation [2] was performed using in-house software and the program Haploview 4.2 [5]. 


\section{Results}

Both affected siblings from the Naples family had mild delay in reaching the child developmental milestones. During the third decade of life, they developed progressive parkinsonism, dystonia, and mild cognitive impairment. Videotapes of these two patients, recorded at the time of our examination, are available (video 1 and video 2). Moreover, the clinical features in the patients of this family and in those of two families previously reported with SYNJ1 mutations are summarized in Table 1.

The first patient (NAPO-41) suffered during adolescence one brief episode of loss of consciousness, which was not otherwise characterized. He developed progressive slowness of movements from the age of 28 years. At our neurological examination, at the age of 31 years, he exhibited hypomimia, monotone and slurred speech, mildly stopped posture, reduced arms swings when walking, oro-mandibular tremor, axial and limb rigidity, bradykinesia, mild trunk dystonia with left flexion, reduced postural reflexes at the pull test, and mild supranuclear upward vertical gaze limitation (this feature being not present during all the examinations). The patient also showed irritability, drooling, and dysphagia. Cerebellar and pyramidal signs were absent. The UPDRS-III score was 42 / 108. The neuropsychological evaluation revealed a normal MMSE score (28/30), but abnormal performances in the tests for verbal and spatial short-term memory, and those for immediate and long-term verbal memory. The FAB score was at the lower limits of the normal range.

His sister (NAPO-42) suffered one episode of generalized tonic-clonic seizures at the age of 16 years. At the age of 26 years, she developed oro-mandibular and left limbs tremor, and subsequently slowness of movements. At our neurological examination, at the age of 27 years, she showed a masked face, monotone and slurred speech, rest tremor in the left limbs and the right arm, limb rigidity, bradykinesia, mildly flexed posture, slow gait with reduced arm swings, and reduced postural reflexes. The knee deep tendon reflexes were brisk. The UPDRS-III score was 32/108. The patient also complained of diffuse muscular pain. The neuropsychological evaluation revealed a low, but still normal MMSE score (25/ 30 ), and abnormal performances in the tests of verbal long and short-term memory. The scores of the Raven's Coloured Progressive Matrices and FAB were below the normal values. The performance at the attentive matrices and phonemic verbal fluency resulted at the lower limits of the normal range.

In both siblings, the blood chemistry, serum ceruloplasmin, ferritin, and copper levels were normal. The Filipin test, performed to exclude the Niemann Pick disease type C, was negative. Levodopa was never administered to these siblings, neither as acute challenge, nor as a chronic treatment. In the affected brother (NAPO-41), the administration of pramipexole up to $3 \mathrm{mg}$ /day resulted in partial improvement

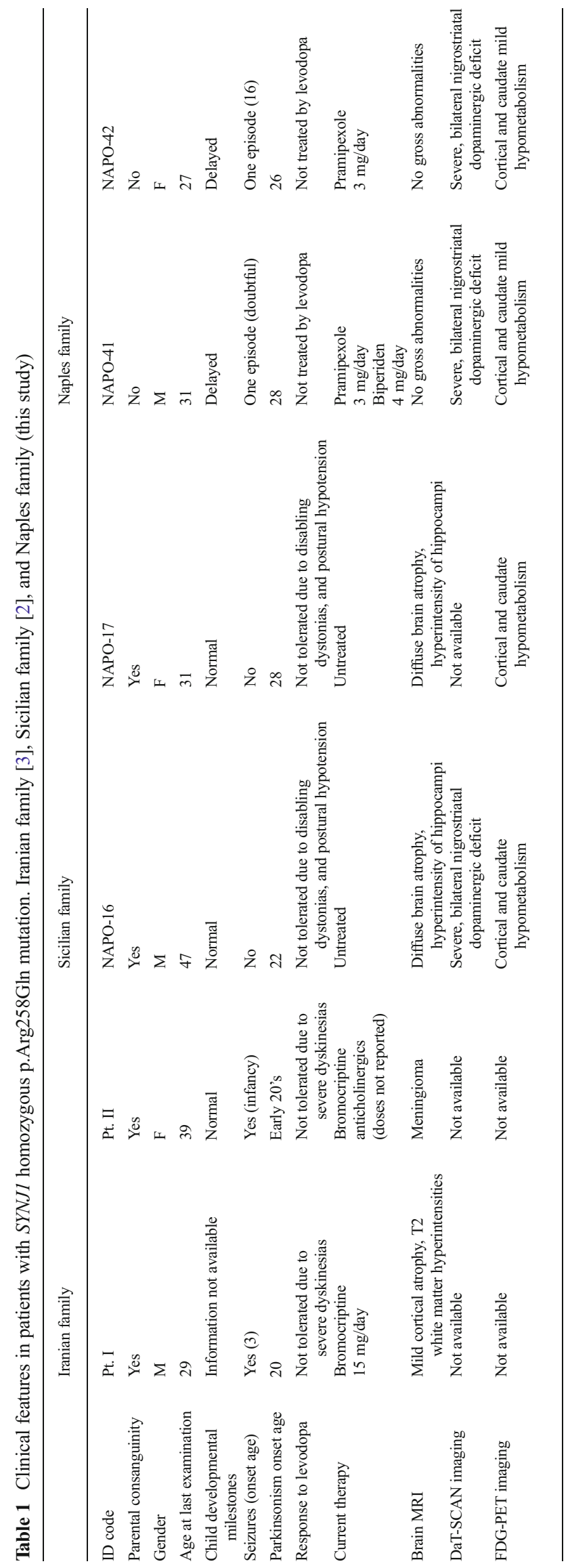


of the parkinsonism signs. Treatment of the affected sister (NAPO-42) with the same dosage of pramipexole and biperiden $2 \mathrm{mg}$ twice a day improved tremor and bradykinesia remarkably. In this patient, muscular pain was successfully treated with duloxetine $30 \mathrm{mg}$ once a day.

SPECT studies revealed in both patients a marked decrease of dopamine transporter density in the whole striatum, which was almost symmetric and more marked in the putamen (Fig. 1d and e). Compared to controls, V3" was reduced by about $75-85 \%$ in the putamen and by $50-70 \%$ in the caudate. FDG-PET studies (Fig. 1a, b, and Suppl. Fig. 1) showed only a very mild relative bilateral hypometabolism in the orbito-basal frontal and temporo-parietal cortices and in the striatum in NAPO-42, and in the caudate in NAPO-41. Brain MRI showed no atrophy or focal abnormalities at the level of the cerebral cortex, basal ganglia, midbrain, and hippocampus (Suppl. Fig. 2). In both patients, the cerebellar dentate nuclei appeared slightly hypointense in the T2-weighted echo-planar images acquired within the diffusion sequence. This finding, which remains of uncertain pathophysiologic significance, was detected also in the patients of the Sicilian family with SYNJ1 mutations, previously reported by us (Suppl. Fig. 3).

The screening of the known genes causing early-onset parkinsonism revealed in both NAPO-41 and NAPO-42 patients a single heterozygous mutation in PARK2 (c.1204C $>\mathrm{T}$, p.Arg402Cys). Genome-wide SNP-based mapping revealed a 2.7-Mb homozygous region on chromosome 21 (flanked by the rs2833664 and rs2834651 markers), which include the SYNJ1 gene (Fig. 2). Sanger sequencing of SYNJ1 exons and exon-intron boundaries revealed a c. $773 \mathrm{G}>\mathrm{A}$ (p.Arg258Gln) mutation, which was homozygous in both affected siblings, and heterozygous in the unaffected parents and one unaffected sib. DNA was not available from the other unaffected sib. Haplotype analysis of the SYNJ1 locus in the patients from this novel family and those from the Sicilian family previously described by us [2] revealed several discordant SNPs, including intragenic SNPs and SNPs in the flanking regions (the minimum distance from the mutation being 4,723 bp) (Fig. 2); these comprise SNPs falling within the same haplotype block of the mutation (Suppl. Fig. 4). This scenario suggests that a common ancestor is unlikely, and the SYNJ1 mutation has arisen independently in the ancestors of these two Italian families.

\section{Discussion}

Here, we report the identification of a third family with earlyonset parkinsonism associated with the same homozygous SYNJ1 mutation. This work supports further that SYNJ1 mutations cause this newly recognized form of neurodegenerative disease (PARK20), but also contributes to the definition of its genetic and clinical features. Our haplotype analysis suggests that a common ancestor is unlikely, and the SYNJ1 p.Arg258Gln mutation has arisen independently in these two
Fig. 1 Neuroimaging findings. Brain metabolic activity using $\left[{ }^{18} \mathrm{~F}\right]$ fluorodeoxyglucose and positron emission tomography (PET) at the level of the basal ganglia in NAPO-41 (a), NAPO-42 (b), and a control subject (c). Slight hypometabolism is evident in the striatum of patient NAPO-42 (b), particularly the caudate nuclei, and in the caudate of patient NAPO-41 (a). Brain dopamine transporter density values (V3") using single photon emission computed tomography (DaTSCAN-SPECT) in NAPO-41 (d) NAPO-42 (e), and a control subject (f). Axial parametric images show a marked presynaptic nigrostriatal dopaminergic deficit in both patients
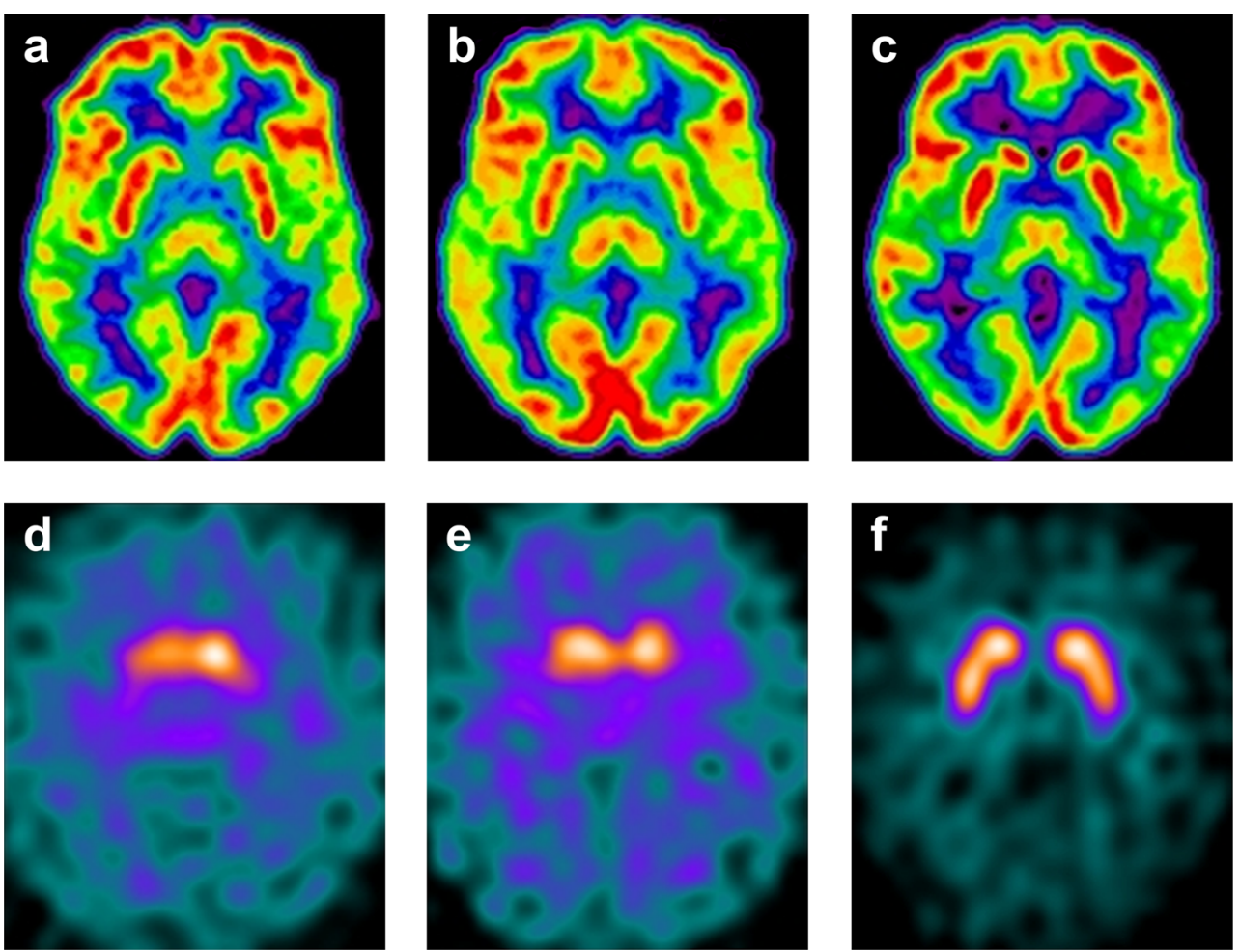


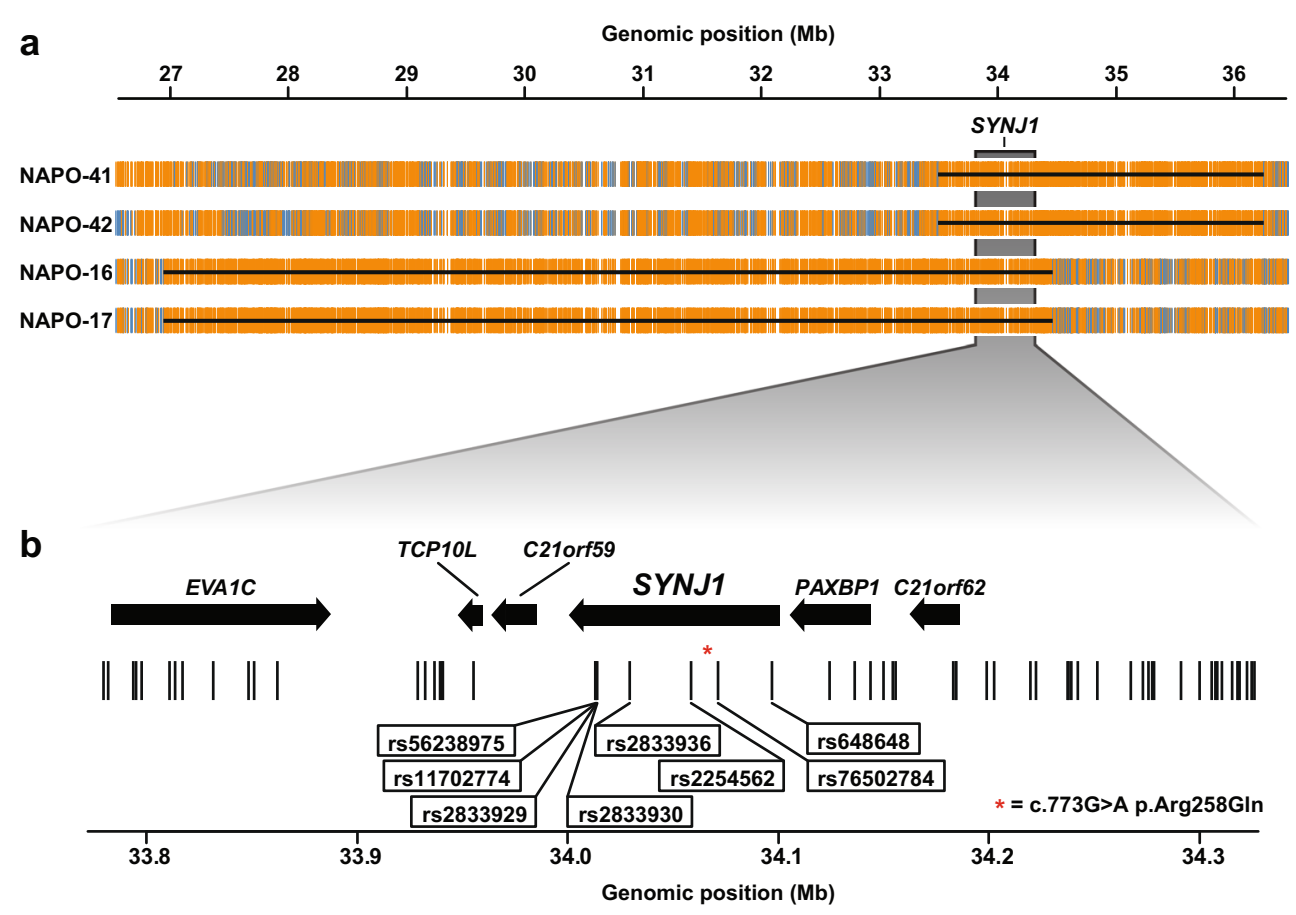

Fig. 2 Homozygosity mapping and haplotype analysis of the SYNJ1 locus. a Homozygosity map of the chromosome 21 in the patients from this family (NAPO-41 and NAPO-42) and in those from the Sicilian family with $S Y N J 1$ mutations previously reported by us (NAPO-16 and NAPO-17) [2]. Homozygous SNPs are depicted in orange, heterozygous SNPs in blue; the position of the SYNJI gene is indicated. The

Italian families. This, in turn, suggests that the guanine in position 773 of the coding region of SYNJ1 is a mutational hotspot. The interpretation of the heterozygous parkin p.Arg402Cys mutation, present in both the Naples siblings with PARK20, remains difficult. We have not identified a second, compound heterozygous mutation in parkin. Furthermore, the pathogenicity of this variant has never been proven and several studies reported it in patients as well as in controls [6-8]. This variant might be a disease-unrelated coincidental finding; however, we cannot exclude that it might have played a role in the disease pathogenesis, together with the primary, causative SYNJI mutations.

In all the PARK20 patients reported so far $(n=6)$, the first symptoms of parkinsonism developed during the third decade of life (Table 1). Atypical signs of various degrees and types have been observed in all these subjects; of note, the response to levodopa was poor, and limited by the development of severe and disabling dyskinesias, dystonias, or postural hypotension. The two patients reported here for the first time have had parkinsonism for only one and three years, respectively, and have not been exposed to levodopa so far, offering therefore a window into the early phases of this newly-identified form of Mendelian neurodegenerative disorder. However, a wide phenotypic variability is emerging, which can only in part be explained by the different disease duration. It is also homozygous regions are highlighted in each patient by horizontal bars. b Haplotype analysis of the $S Y N J 1$ locus reveals several discordant SNPS between the two families with the SYNJ1 mutation. Only discordant SNPs are shown; for the SNPs located within the SYNJ1 gene, RS codes are shown; the position of the c.773G $>$ A (p.Arg258Gln) mutation is indicated with a red asterisk

likely that the identification of additional patients with this form will further expand the phenotypic and mutational spectra.

From the clinical standpoint, PARK20 must be considered in the diagnostic work-up of patients with early-onset atypical parkinsonism. Of note, seizures have been reported in some of these patients (Table 1), as well as in some with PARK19, another recently identified form of early-onset parkinsonism, caused by mutations in the gene DNAJC6 (OMIM 608375) The protein product of this latter gene, auxilin-1, is a direct functional partner of synaptojanin-1 during the process of uncoating of the synaptic vesicles. The presence of seizures might therefore be a red flag for suspecting one of these two forms (PARK19, PARK20).

The identification of SYNJ1 mutations in PARK20 points to defective post-endocytic recycling of synaptic vesicles in the pathogenesis of neurodegeneration. Abnormalities at the same level have been implicated in other forms of genetic parkinsonism, such as those caused by mutations in LRRK2 [9], parkin [10], SNCA [11, 12], VPS35 [13, 14], and DNAJC6 $[15,16]$, suggesting that an abnormal recycling of synaptic vesicles is a central theme in the pathogenesis. Therefore, elucidating further the molecular mechanisms of PARK20 might also foster our understanding of the more common forms of Parkinson's disease. 
Disclosure This study was supported by grants from the Stichting ParkinsonFonds (The Netherlands) to V.B. The authors declare that they have no conflict of interest. All experiments performed in this study comply with the current laws of the countries in which they were performed.

\section{References}

1. Bonifati V (2014) Genetics of Parkinson's disease - state of the art, 2013. Parkinsonism Relat Disord 20(Suppl 1):S23-S28. doi:10. 1016/S1353-8020(13)70009-9

2. Quadri M, Fang M, Picillo M, Olgiati S, Breedveld GJ, Graafland J, Wu B, Xu F, Erro R, Amboni M, Pappata S, Quarantelli M, Annesi G, Quattrone A, Chien HF, Barbosa ER, International Parkinsonism Genetics N, Oostra BA, Barone P, Wang J, Bonifati V (2013) Mutation in the SYNJ1 gene associated with autosomal recessive, early-onset Parkinsonism. Hum Mutat 34(9):1208-1215. doi:10. 1002/humu.22373

3. Krebs CE, Karkheiran S, Powell JC, Cao M, Makarov V, Darvish H, Di Paolo G, Walker RH, Shahidi GA, Buxbaum JD, De Camilli P, Yue Z, Paisan-Ruiz C (2013) The Sac1 domain of SYNJ1 identified mutated in a family with early-onset progressive Parkinsonism with generalized seizures. Hum Mutat 34(9):1200-1207. doi:10.1002/ humu. 22372

4. Varrone A, Salvatore E, De Michele G, Barone P, Sansone V, Pellecchia MT, Castaldo I, Coppola G, Brunetti A, Salvatore M, Pappata S, Filla A (2004) Reduced striatal [123 I]FP-CIT binding in SCA2 patients without parkinsonism. Ann Neurol 55(3):426-430. doi:10.1002/ana.20054

5. Barrett JC, Fry B, Maller J, Daly MJ (2005) Haploview: analysis and visualization of LD and haplotype maps. Bioinformatics 21(2):263265. doi:10.1093/bioinformatics/bth457

6. Bertoli-Avella AM, Giroud-Benitez JL, Akyol A, Barbosa E, Schaap O, van der Linde HC, Martignoni E, Lopiano L, Lamberti P, Fincati E, Antonini A, Stocchi F, Montagna P, Squitieri F, Marini P, Abbruzzese G, Fabbrini G, Marconi R, Dalla Libera A, Trianni G, Guidi M, De Gaetano A, Boff Maegawa G, De Leo A, Gallai V, de Rosa G, Vanacore N, Meco G, van Duijn CM, Oostra BA, Heutink P, Bonifati V (2005) Novel parkin mutations detected in patients with early-onset Parkinson's disease. Mov Disord 20(4):424-431. doi:10. 1002/mds.20343

7. Bruggemann N, Mitterer M, Lanthaler AJ, Djarmati A, Hagenah J, Wiegers K, Winkler S, Pawlack H, Lohnau T, Pramstaller PP, Klein C, Lohmann K (2009) Frequency of heterozygous Parkin mutations in healthy subjects: need for careful prospective follow-up examination of mutation carriers. Parkinsonism Relat Disord 15(6):425-429. doi:10.1016/j.parkreldis.2008.11.014

8. Brooks J, Ding J, Simon-Sanchez J, Paisan-Ruiz C, Singleton AB, Scholz SW (2009) Parkin and PINK1 mutations in early-onset
Parkinson's disease: comprehensive screening in publicly available cases and control. J Med Genet 46(6):375-381. doi:10.1136/jmg. 2008.063917

9. Matta S, Van Kolen K, da Cunha R, van den Bogaart G, Mandemakers W, Miskiewicz K, De Bock PJ, Morais VA, Vilain S, Haddad D, Delbroek L, Swerts J, Chavez-Gutierrez L, Esposito G, Daneels G, Karran E, Holt M, Gevaert K, Moechars DW, De Strooper B, Verstreken P (2012) LRRK2 controls an EndoA phosphorylation cycle in synaptic endocytosis. Neuron 75(6):1008-1021. doi:10.1016/j.neuron.2012.08.022

10. Trempe JF, Chen CX, Grenier K, Camacho EM, Kozlov G, McPherson PS, Gehring K, Fon EA (2009) SH3 domains from a subset of BAR proteins define a Ubl-binding domain and implicate parkin in synaptic ubiquitination. Mol Cell 36(6):1034-1047. doi:10. 1016/j.molcel.2009.11.021

11. Ben Gedalya T, Loeb V, Israeli E, Altschuler Y, Selkoe DJ, Sharon R (2009) Alpha-synuclein and polyunsaturated fatty acids promote clathrin-mediated endocytosis and synaptic vesicle recycling. Traffic 10(2):218-234. doi:10.1111/j.1600-0854.2008.00853.x

12. Nemani VM, Lu W, Berge V, Nakamura K, Onoa B, Lee MK, Chaudhry FA, Nicoll RA, Edwards RH (2010) Increased expression of alpha-synuclein reduces neurotransmitter release by inhibiting synaptic vesicle reclustering after endocytosis. Neuron 65(1):66-79. doi:10.1016/j.neuron.2009.12.023

13. Vilarino-Guell C, Wider C, Ross OA, Dachsel JC, Kachergus JM, Lincoln SJ, Soto-Ortolaza AI, Cobb SA, Wilhoite GJ, Bacon JA, Behrouz B, Melrose HL, Hentati E, Puschmann A, Evans DM, Conibear E, Wasserman WW, Aasly JO, Burkhard PR, Djaldetti R, Ghika J, Hentati F, Krygowska-Wajs A, Lynch T, Melamed E, Rajput A, Rajput AH, Solida A, Wu RM, Uitti RJ, Wszolek ZK, Vingerhoets F, Farrer MJ (2011) VPS35 mutations in Parkinson disease. Am J Hum Genet 89(1):162-167. doi:10.1016/j.ajhg.2011.06.001

14. Zimprich A, Benet-Pages A, Struhal W, Graf E, Eck SH, Offman MN, Haubenberger D, Spielberger S, Schulte EC, Lichtner P, Rossle SC, Klopp N, Wolf E, Seppi K, Pirker W, Presslauer S, Mollenhauer B, Katzenschlager R, Foki T, Hotzy C, Reinthaler E, Harutyunyan A, Kralovics R, Peters A, Zimprich F, Brucke T, Poewe W, Auff E, Trenkwalder C, Rost B, Ransmayr G, Winkelmann J, Meitinger T, Strom TM (2011) A mutation in VPS35, encoding a subunit of the retromer complex, causes late-onset Parkinson disease. Am J Hum Genet 89(1):168-175. doi:10.1016/j.ajhg. 2011.06.008

15. Edvardson S, Cinnamon Y, Ta-Shma A, Shaag A, Yim YI, Zenvirt S, Jalas C, Lesage S, Brice A, Taraboulos A, Kaestner KH, Greene LE, Elpeleg O (2012) A deleterious mutation in DNAJC6 encoding the neuronal-specific clathrin-uncoating co-chaperone auxilin, is associated with juvenile parkinsonism. PLoS One 7(5):e36458. doi:10. 1371/journal.pone.0036458

16. Koroglu C, Baysal L, Cetinkaya M, Karasoy H, Tolun A (2013) DNAJC6 is responsible for juvenile parkinsonism with phenotypic variability. Parkinsonism Relat Disord 19(3):320-324. doi:10.1016/j. parkreldis.2012.11.006 